\title{
Tensiones historiográficas en las narrativas del arte contemporáneo: Desconexiones entre la historia de las artes electrónicas y el circuito del arte contemporáneo hegemónico
}

Jazmín Adler

Consejo Nacional de Investigaciones Científicas y Técnicas (CONICET) jazminadler@gmail.com

Fecha recepción: 21.06.2019 / Fecha aceptación: 06.11.2019

\section{Resumen}

El presente artículo analiza los modos en que la historiografía del arte contemporáneo hegemónico ha discurrido de manera paralela a los devenires de las artes electrónicas, al punto de que las narrativas de la historia del arte contemporáneo canónico no han tendido a incluir artistas, obras y prácticas

\section{Abstract}

This article analyses the ways in which mainstream contemporary art historiography has largely ignored the development of electronic art. Thus, dominant contemporary art narratives have tended not to include artists, artworks or practices engaged in technological experi-

\footnotetext{
*Este artículo constituye una adaptación de la primera parte del Capítulo Dos de la tesis correspondiente al Doctorado en Teoría Comparada de las Artes, titulada Ruinología contemporánea: emergencias de la escena de las poéticas electrónicas en Buenos Aires. La tesis fue defendida el 10 de julio de 2018 en la Universidad Nacional de Tres de Febrero (Buenos Aires, Argentina) y financiada por la beca doctoral del Consejo Nacional de Investigaciones Científicas y Técnicas (CONICET), bajo dirección de la Dra. Claudia Kozak y la Dra. Mariela Yeregui.
} 
Miscelánea | Tensiones historiográficas en las narrativas del arte contemporáneo

abocados a la experimentación tecnológica. Luego de indagar en las diferentes contribuciones teóricas que conforman el estado de la cuestión sobre el objeto de estudio, el artículo propone estrategias críticas y fundamentos conceptuales destinados a establecer puntos de conexión entre la historia de ambos circuitos.

\section{Palabras clave}

arte contemporáneo, artes electrónicas, contemporaneidad, nuevos medios, tecnologías mentation. After exploring different theories that constitute the state of the art in relation to the subject under study, I shall suggest critical strategies and conceptual groundings aimed at establishing connections between the histories of both fields.

\section{Keywords}

Contemporary art, contemporaneity, electronic arts, new media, technologies 


\section{Introducción}

Durante los últimos años, diversos críticos y académicos han participado de un debate candente en torno al lugar que las poéticas electrónicas ${ }^{1}$ ocupan en el mundo del arte contemporáneo, así como la naturaleza de las relaciones establecidas entre ambas escenas. Uno de los autores más activos al respecto es Edward Shanken, cuyo análisis replica que el desarrollo sostenido del circuito del arte contemporáneo hegemónico (mainstream contemporary art) desde mediados de los años noventa coincidió temporalmente con el crecimiento del arte de los nuevos medios (new media art), fenómeno que habría provocado el advenimiento de discursos divergentes:

Desde mediados de la década del noventa, el arte de los nuevos medios devino una importante fuerza para el desarrollo económico y cultural, estableciendo sus propias instituciones. La investigación colaborativa y transdisciplinaria en la intersección del arte, la ciencia y la tecnología también ganó estima y apoyo institucional con los programas de doctorado interdisciplinarios que fueron proliferando alrededor del mundo. Durante el mismo período, el arte contemporáneo hegemónico experimentó un crecimiento dramático en su mercado y su popularidad, impulsado por la prosperidad económica y la propagación de museos, ferias de arte y exposiciones internacionales. Este entorno dinámico nutrió una enorme creatividad e invención en el trabajo de artistas, curadores, teóricos y pedagogos que trabajan en ambas escenas. Sin embargo, raramente el arte contemporáneo hegemónico converge con el mundo del arte de los nuevos medios. Como resultado, los discursos han resultado cada vez más divergentes. ${ }^{2}$

1. Al referir a las poéticas electrónicas, aludimos a aquellas obras que hacen uso material, formal, estético y conceptual de las tecnologías electrónicas, ya sea analógicas o digitales, en distintas instancias del proceso creativo. Se trata de una escena integrada por prácticas diversas, como proyectos de net art, CD-ROM interactivos, fotografías intervenidas digitalmente, videoarte, entornos sensoriales, instalaciones interactivas, esculturas robóticas, investigaciones en el terreno del diseño paramétrico, impresión 3D, arte generativo y vida artificial, entre otras manifestaciones.

2. E. Shanken, “Contemporary Art and New Media: Digital Divide or Hybrid Discourse?", Art Research Journal / Revista de Pesquisa em Arte, 2, 2015, 75-98, 75. Traducción propia. A partir de aquí, cuando las citas se encuentran en otro idioma, la traducción al español es incluida en el cuerpo del texto y en la nota al pie, junto a la referencia bibliográfica, aquellas son transcritas en su idioma original: «Since the mid-1990s, new media art (NMA) has become an important force for economic and cultural development interna- 
Podemos incluso retrotraer los postulados de Shanken hacia los comienzos del arte contemporáneo. Cuando en las primeras líneas del famoso artículo dedicado a la expansión del campo escultórico, originalmente publicado en 1979, Rosalind Krauss asevera que «en los últimos años una serie de cosas bastante sorprendentes han recibido el nombre de esculturas $»^{3}$, la autora enumera fotografías que documentan espejos ubicados en habitaciones comunes, líneas dibujadas en el desierto estadounidense y pasillos con televisores, como Live-Taped Video Corridor de Bruce Nauman. Excepto por la alusión a la fotografía y la videoinstalación, Krauss omite mencionar a una serie de trabajos que también la habrían dejado atónita. Lo cierto es que mientras se difundían las obras del minimalismo, el arte conceptual, el Land Art y otras de las manifestaciones descritas por Krauss, grandes referentes de la confluencia entre el arte y la tecnología -Ben Laposky, Nicolas Schöffer, Gyula Kosice, Lucio Fontana, Michael Noll, Bela Julesz, Charles Csuri, Frieder Nake, Georg Nees, Wen Ying Tsai, Nam June Paik, entre muchos otros- dilataban los límites del campo artístico hasta entonces conocido, a través de esculturas robóticas, instalaciones interactivas y obras realizadas por computadora.

A lo largo del presente artículo, argumentaremos que las historias de ambos circuitos fueron desarrolladas en paralelo, es decir que sus respectivos devenires han demostrado escasos puntos de convergencia. De hecho, los sistemas de producción, crítica, difusión y enseñanza de la escena de las poéticas electrónicas fueron fortaleciéndose a partir de los años sesenta y, desde entonces, impulsaron la conformación de una escena autónoma, provista de sus propios espacios de exhibición, publicaciones, carreras universitarias, críticos, curadores, festivales, encuentros, congresos y otros ámbitos de investigación y exposición, tales como el ZKM en Alemania, el InterCommunication Center en Tokyo, Ars Electronica en Linz, el International Symposium on the Electronic Art -más conocido como ISEA- y el Banff Centre en Canadá, solo por mencionar algunos. Por su parte, como argüiremos a continuación, las historiografías del arte contemporáneo dominantes no han producido narrativas críticas que permitan establecer conexiones entre las creaciones de aquellos artistas que emplean las tecnologías en sus obras, prácticas y procesos, y quienes no incorporan medios tecnológicos de manera preeminente.

tionally, establishing its own institutions. Collaborative, transdisciplinary research at the intersections of art, science, and technology also has gained esteem and institutional support with interdisciplinary Ph.D. programs proliferating around the world. During the same period, mainstream contemporary art (MCA) experienced dramatic growth in its market and popularity, propelled by economic prosperity and the propagation of international museums, art fairs and exhibitions. This dynamic environment has nurtured tremendous creativity and invention by artists, curators, theorists and pedagogues operating in both domains. Yet rarely does the mainstream art world converge with the new media art world. As a result, their discourses have become increasingly divergent.»

3. R. Krauss, "La escultura en el campo expandido", en H. Foster (coord.), La posmodernidad, Barcelona, 2002, 59. 


\section{Desconexiones historiográficas entre las poéticas electrónicas y el arte contemporáneo hegemónico}

En efecto, entre las décadas del sesenta y setenta, en concomitancia con la irrupción del arte contemporáneo, advinieron una serie de tendencias artísticas que socavaron los mundos del arte instituidos, un fenómeno que finalmente terminó desterrando a los artistas atraídos por la experimentación tecnológica a una suerte de nicho separado del ámbito del arte contemporáneo. Adscribiendo a la teoría de Howard Becker plasmada en Los mundos del $a_{r t e}{ }^{4}$, Domenico Quaranta retomó la metáfora introducida por Tom Wolfe en La palabra pintada $a^{5}$ para aprehender el vínculo establecido entre los movimientos de vanguardia y el establishment del arte. Wolfe describe aquella relación como un "ritual de apareamiento», desarrollado en dos etapas. En la primera -«Danza de los bohemios» (The Boho Dance)- los artistas muestran su obra innovadora, realizada por fuera del arte establecido. En la segunda instancia, denominada «Consumación» (The Consummation), el establishment recluta a nuevos artistas y movimientos de la bohemia, transformándolos en figuras célebres del mundo del arte. El galanteo entre las poéticas electrónicas y el arte contemporáneo hegemónico nunca habría alcanzado la segunda fase. Siguiendo los postulados de Quaranta, se trataría de un baile entre dos amantes que coquetean pero cuya relación nunca se llega a consumar ${ }^{6}$.

La idea de que las poéticas electrónicas fueron consolidándose como un nicho aislado con respecto al arte contemporáneo fue también analizada por Geert Lovink. El teórico holandés sugirió que aquellas no lograron expandirse por fuera de la subcultura que conforman, al punto de constituirse como un campo independiente, fundado en torno a exposiciones, festivales, reuniones académicas y publicaciones especializadas, pero todavía relativamente separado de otras prácticas artísticas contemporáneas. Aunque vivimos en una época signada por la expansión de Internet, teléfonos celulares y diversas tecnologías que circulan cotidianamente en las sociedades de nuestros tiempos, hace diez años todavía las artes electrónicas parecían actuar en un gueto autorreferencial dominado por un «tecnofetichismo» ${ }^{7}$.

La desconexión historiográfica entre ambas escenas se percibe, por ejemplo, al ponderar la desatención de teóricos canónicos del arte contemporáneo, como Rosalind Krauss o Charles Harrison, hacia las reflexiones de Jack Burnham sobre el campo escultórico. En sus investigaciones sobre el arte conceptual y la historia de la escultura moderna, los autores parecen desconocer los postulados del escritor inglés, cuyos libros Beyond Modern Sculpture: The Effects of Science and Technology on the Sculpture of Our Time (1968), The Structure of Art (1971) o The Great Western Salt Works (1973), compilación de artículos publicados en Arts Magazine y Artforum en los años precedentes, abordan temas afines a los estudios de Krauss y Harrison:

4. H. Becker, Los mundos del arte. Sociología del trabajo artístico, Bernal, 2008.

5. T. Wolfe, La palabra pintada, Barcelona, 1975.

6. D. Quaranta, Beyond New New Media Art, Brescia, 2013, 133.

7. G. Lovink, "New Media: In Search of The Cool Obscure". En línea en: http://bampfa.berkeley.edu/media/lovink.mp4. [Consulta: 31.05.19] 
En lugar de dignificar las teorías artísticas de Burnham disintiendo con él de manera directa, Harrison eludió referir explícitamente a él. Al igual que la teórica y crítica americana Rosalind Krauss, esta exclusión contribuyó con una agenda crítica en revistas de arte influyentes que han minimizado las contribuciones de Burnham hacia la historia del arte. Passages in Modern Sculpture de Krauss no incluyó Beyond Modern Sculpture en su bibliografía. ${ }^{8}$

En realidad, si bien en Pasajes en la escultura moderna, Krauss ${ }^{9}$ sí hace mención de Beyond Modern Sculpture de Burnham cuando recupera determinadas obras que hicieron uso de las tecnologías, por ejemplo, Modulador de espacio-luz de Moholy-Nagy, las citas apuntan a criticar la tesis de Burnham, de acuerdo a la cual la ambición fundamental de la escultura habría sido desde sus inicios la reproducción de la vida, mientras que la expansión de las nuevas tecnologías permitiría a largo plazo asimilarla a la cibernética. Krauss cuestiona esta idea argumentando que muchas obras nunca tuvieron una intención mimética, como los readymades duchampianos, las construcciones de Picasso, o bien el proyecto de Tatlin para el monumento a la Tercera Internacional. No obstante, el «futuro de metas fáusticas ${ }^{10}$ que vislumbra en los postulados de Burnham conduce a la autora a derivar conclusiones simplistas y apresuradas:

El libro de Burnham es una de las exposiciones más amplias y minuciosas de la escultura puesta al servicio de una concepción mecanicista del mundo. Pero, lejos de ser necesaria, esa concepción es precisamente contra lo que gran parte de la escultura contemporánea (y del arte en general) quiere luchar. ${ }^{11}$

Es seguro que por momentos la concepción teleológica de Burnham acerca del desarrollo de la escultura insinúa que los medios tecnológicos pueden ser utilizados de manera neutral, descuidando que los modos en que éstos son empleados implican determinados posicionamientos socio-políticos. No obstante, la dura sentencia de Krauss olvida leer al trabajo de Burnham en sintonía con las transformaciones operadas en la escultura contemporánea no necesariamente tecnológica, en el marco del proceso de desmaterialización que empezaba a caracterizar a las obras producidas en los años sesenta y la irrupción del arte conceptual. Pamela Lee ${ }^{12}$ sostiene que los fenómenos referidos por muchos críticos de la época como

8. E. Shanken, Art in the Information Age: Cybernetics, Software, Telematics and the Conceptual Contributions of Art and Technology to Art History and Aesthetic Theory, North Carolina, 2001, 159. Traducción propia. «Rather than dignify Burnham's theories of art by disagreeing with them directly, Harrison avoided specific reference to him. Like American critic and historian Rosalind Krauss, this exclusion contributed to a critical agenda in influential art journals that has minimized Burnham's contributions to art history. Krauss's Passages in Modern Sculpture did not include Beyond Modern Sculpture in its bibliography».

9. R. Krauss, Pasajes en la escultura moderna, Madrid, 2002, 207.

10. R. Krauss, Pasajes en la..., op. cit., 209.

11. R. Krauss, Pasajes en la..., op. cit., 210.

12. P. Lee, Chronophobia: On Time in the Art of the 1960s, Cambridge, 2004, 240. 
«desmaterialización del objeto artístico», concepto sobre el cual volveremos en el próximo apartado, y "post-formalismo», eran descritos por Burnham según la retórica de la comunicación y las nuevas tecnologías. Aparentemente, Krauss y otros críticos contemporáneos no concibieron dichas ligazones.

\section{La noción de «desmaterialización» en las inmediaciones de historiografías divergentes}

La materialidad de las obras pareciera ser un aspecto central para indagar en la divergencia historiográfica entre ambas escenas. Cierto desinterés del arte contemporáneo hegemónico hacia las obras tecnológicas radicaría en la materialidad de estos proyectos, basados en dispositivos y artefactos que frecuentemente obstaculizan la comprensión de sus dimensiones conceptuales. Por lo tanto, hasta que el mercado no termine por acoger a estas prácticas, la historia del arte electrónico no será reconocida por el arte contemporáneo dominante ${ }^{13}$. Como Patrick Lichty discute en su respuesta al polémico artículo de Claire Bishop, titulado «Digital Divide» ${ }^{14}$, la supervivencia del sistema de las artes visuales, cuyo apego a los objetos coleccionables es aún primordial, se siente amenazado por la desmaterialización que introduce la revolución digital ${ }^{15}$, idea que de cierto modo es reconocida por la propia Bishop: «en la situación más utópica, la revolución digital abre una nueva realidad de cultura colectiva, desmaterializada, desprovista de autores y con dificultades para ingresar al mercado; en el peor caso, da signos de la inminente obsolescencia de las artes visuales en sí mismas» ${ }^{16}$.

Uno de los aspectos que subraya Bishop en el artículo citado es la práctica ausencia de reflexión crítica acerca de las tecnologías digitales empleadas por las obras. Según su perspectiva, son pocos los proyectos que tematizan o reflejan profundamente cómo experimentamos

13. E. Shanken, Inventar el futuro. Arte, Electricidad, Nuevos Medios. Nueva York, 2013, 116.

14. C. Bishop, "Digital Divide. Claire Bishop on Contemporary Art and New Media”, en Artforum, 2012, 436-442. El artículo de Claire Bishop fue publicado en septiembre de 2012 en Artforum. Ha sido incluido en el número "Art's New Media”, editado por Michelle Kuo con motivo del decimoquinto aniversario de esta revista. Allí Bishop sostiene que el «arte de los nuevos medios» (new media art) constituye un campo especializado que raramente se superpone con el circuito del arte contemporáneo hegemónico, identificado con las galerías comerciales, el Premio Turner y los pabellones nacionales de la Bienal de Venecia. Este es el motivo por el cual la autora opta por estudiar el impacto de las tecnologías digitales en la obra de una serie de artistas del mundo del arte contemporáneo mainstream, en lugar de examinar proyectos realizados por artistas del mundo de las artes electrónicas. El ensayo de Bishop ha sido cuestionado por varios autores. Una de las críticas más duras fue la de Patrick Lichty (2013), quien caracterizó a la postura de Bishop como tradicionalista y reactiva.

15. P. Lichty, "A Disjointed Conversation - Claire Bishop. The Digital Divide and the State of New Media Contemporary Art”. En línea en: http://rhizome.org/community/44886/. [Consulta: 01.06.19]

16. Bishop, “Digital Divide...”, op. cit., 441. Traducción propia. «At its most utopian, the digital revolution opens up a new dematerialized, deauthored, and unmarketable reality of collective culture; at its worst, it signals the impending obsolescence of visual art itself». 
y somos alterados por la digitalización de nuestra existencia. No solo podemos objetar esta afirmación -el propio Quaranta proporcionó ejemplos acertados de artistas que exploran la especificidad del medio de modos diversos ${ }^{17}$-, sino además arriesgar que la tesis de Bishop comete el error de suponer que las artes electrónicas deberían explícitamente hacer referencia a las tecnologías involucradas, cuando a otras prácticas contemporáneas no se les exige la tematización o el reflejo de los medios y herramientas de los cuales se valen. Más aun, cuando Bishop refiere a la desmaterialización como una cualidad distintiva de la revolución digital, no considera que la noción de obra desmaterializada se remonta por lo menos a los inicios del arte contemporáneo, en la medida en que el concepto de desmaterialización no alude a la ausencia de materiales ni niega la existencia del aspecto material de las obras. Por el contrario, remite a la emergencia de materialidades que hasta entonces no formaban parte del ámbito de las artes. De allí que Lucy Lippard explicara en Seis años: la desmaterialización del objeto artístico de 1966 a 1972, un texto dedicado al análisis del arte conceptual reciente, que la desmaterialización es interpretada como una «retirada del énfasis sobre los aspectos materiales (singularidad, permanencia, atractivo decorativo) $»^{18}$, vale decir, una pérdida de protagonismo del aspecto material, estético y formal de la obra. Ya en 1967, junto con John Chandler, Lippard había escrito el artículo "The Dematerialization of Art", publicado en febrero de 1968 en Art International. Los autores allí sostenían:

Durante los años sesenta, el proceso anti-intelectual, emocional/intuitivo del quehacer artístico, característico de las últimas dos décadas, comenzó a dar lugar a un arte ultra-conceptual que enfatiza el proceso de pensamiento de manera casi exclusiva (...) Esta tendencia parece estar provocando una profunda desmaterialización del arte, especialmente del arte como objeto y, si continúa prevaleciendo, puede resultar en la obsolescencia total del objeto. ${ }^{19}$

La hipótesis de que la noción de desmaterialización asimismo opera en otras obras que no recurren a medios tecnológicos ha sido el nudo gordiano de la curaduría de Jean-François Lyotard en Les Immatériaux, una exposición organizada en el Centro Georges Pompidou entre marzo y julio de 1985. Por inmateriales Lyotard comprendía la emergencia de nuevos materiales y materialidades devenidos de la expansión de las tecnologías telecomunicacionales, los cuales comenzaban a transformar la sensibilidad y generaban una ruptura con respecto a la concepción moderna de materialidad asociada a obras, objetos y cuerpos asibles. Sin embargo, la exposición no se limitaba a difundir las obras que resultaban de la experimen-

17. Quaranta, Beyond..., op. cit., 12.

18. L. Lippard, Seis años: la desmaterialización del objeto artístico de 1966 a 1972, Madrid, 2004, 33.

19. J. Chandler, J. y L. Lippard, “The Dematerialization of Art”, Art International, 1968, 31-36, 31. Traducción propia. «During the 1960's, the anti-intellectual, emotional/intuitive processes of art-making characteristic of the last two decades have begun to give way to an ultra-conceptual art that emphasizes the thinking process almost exclusively (...) Such a trend appears to be provoking a profound dematerialization of art, especially of art as object, and if it continues to prevail, it may result in the object's becoming wholly obsolete». 
tación con los nuevos medios (imágenes computarizadas, hologramas y piezas lumínicas), sino también trabajos futuristas, constructivistas y posimpresionistas, entre otras creaciones realizadas en soportes y lenguajes tradicionales ${ }^{20}$. Las diferentes obras convivían en la muestra ideada por Lyotard, dado que todas ellas, desde distintos ángulos, lanzaban interrogantes sobre los cinco ejes conceptuales que organizaban la curaduría:

¿De dónde vienen los mensajes que nos son propuestos (cuál es su maternidad)?, ¿A qué se refieren estos mensajes (a qué materia se adscriben)?, ¿Según qué códigos son descifrables (cuál es su matriz)?, ¿Sobre qué soporte son inscritos (cuál es su material)?, ¿Cómo son transmitidos a sus destinatarios (cuál es la materialidad de esta dinámica)? Estas secuencias son ilustradas por objetos provenientes de ámbitos heterogéneos (puntura, biología, fotografía, arquitectura, astrofísica, música, etc.), reagrupadas de acuerdo con el régimen de una pregunta única que ilumina un aspecto de la complejidad. ${ }^{21}$

En una línea de análisis semejante a la de Lyotard, Ana Longoni sustentó, tres décadas más tarde, que la desmaterialización acontecida en los años sesenta no constituyó la ausencia total de materiales, sino la experimentación con nuevos materiales excluidos del campo artístico, como personas, desechos y diferentes disciplinas científicas ${ }^{22}$. Por su parte, Claudia Kozak argumentó que el proceso de desmaterialización significó, además de la investigación con materialidades difíciles de ser percibidas sensorialmente por carecer de existencia concreta -como es el caso de la materialidad de la información-, la puesta en práctica de otras materialidades heterodoxas que reemplazan a los materiales nobles tradicionalmente trabajados y elaborados mediante la experticia de la mano del artista ${ }^{23}$.

Los postulados de ambas autoras evidencian que el concepto de desmaterialización no supone un rasgo privativo de las artes tecnológicas, ni constituye un atributo específicamente devenido de la revolución digital como sugirió Bishop, sino uno de los aspectos característicos de la expansión propia del arte contemporáneo en su afán de forjar una nueva concepción de obra que trascienda ampliamente las fronteras del campo artístico instituido. Un enfoque teórico de esta naturaleza permite reformular la historiografía

20. Algunos de los artistas que formaron parte de la exhibición fueron Giacomo Balla, Sonia Delaunay, Yves Klein, Lucio Fontana, Piero Manzoni, Joseph Kosuth, Dan Flavin, Vito Acconci, Dan Graham, László Moholy-Nagy, Stephen Benton, Kazimir Malévich, Andy Warhol y Marcel Duchamp.

21. J-F. Lyotard, Les Immatériaux, Conferencia de prensa, 8 de enero de 1985, Centro Georges Pompidou, 2. Traducción propia. «D’où viennent les messages qui nous sont proposés (quelle est leur maternité)? à quoi se réfèrent-ils (à quelle matière se rapportent-ils) ? Selon quel code sont-ils déchiffrables (quelle en est la matrice) ? Sur quel support sont-ils inscrits (quel est leur matériau) ? Comment sont-ils transmis aux destinataires (quel est le matériel de cette dynamique) ? Ces séquences sont illustrées par des objets empruntés à des domaines hétérogènes (peinture, biologie, photographie, architecture, astrophysique, musique... etc) regroupés sous le régime d'une question unique, qui en éclaire un aspect de la complexité.»

22. A. Longoni, Oscar Masotta. Revolución en el arte, Buenos Aires, 2017.

23. C. Kozak, "Literatura digital y materialidad. Cómo se lee", en P. Alsina y A. Rodríguez Granell (coords.), “Art Matters”, Artnodes, 15, 2015, 90-98. 
del arte contemporáneo de manera que efectivamente coexistan expresiones del proceso de desmaterialización tecnológica - píxeles, proyecciones, algoritmos, efectos lumínicos y comportamientos cinéticos- y otras manifestaciones contemporáneas -obras conceptuales, arte povera, Land Art, solo por mencionar algunos ejemplos- las cuales, sin recurrir a las tecnologías, también propusieron desmaterializar las obras mediante la puesta en jaque de la idea de obra material, física, tangible, permanente y cerrada, predominante a lo largo de la historia de las artes visuales en Occidente.

\section{Tentativas de reescritura: reformulaciones de la historia del arte contemporáneo dominante}

Las tensiones conceptuales repuestas en las páginas precedentes incitan a proponer nuevas historiografías del arte contemporáneo, a través de la configuración de un discurso híbrido que pueda tender puentes entre las historias de cada escena ${ }^{24}$. El llamado de Shanken a fundar nuevas perspectivas que permitan repensar los puntos de contacto entre las poéticas electrónicas y la escena hegemónica fue plasmado en su ensayo «Art in the Information Age: Technology and Conceptual Art» ${ }^{25}$. En este escrito el autor propone revisar las relaciones entre el arte conceptual y obras tecnológicas que no han sido incorporadas en los relatos canónicos sobre el conceptualismo, si bien ambas constituirían manifestaciones anticipadas de la denominada «era de la información» ${ }^{26}$. De manera análoga, su libro Art and Electronic Media, editado por Phaidon en 2009 como parte de la colección Themes and Movements, plantea un abordaje heterodoxo. Mientras que cada uno de los volúmenes que integran la serie están dedicados a diferentes tendencias y movimientos artísticos (minimalismo, arte conceptual, Land Art, arte povera, dadaísmo, surrealismo y pop, entre otros), Shanken disputó aquellas fronteras tradicionales estructurando su trabajo en función de temáticas que no son cronológicas pero tampoco se basan en los medios empleados por los proyectos, sino que apuntan a develar continuidades entre distintas épocas y géneros: «(...) procedo a derribar los sistemas de valoración basados en el mercado al unir perfectamente a artistas contemporáneos de primera categoría como Bruce Nauman, Jenny Holzer y Olafur Eliasson con grandes figuras del arte de los nuevos medios como Roy Ascott, Lynn Hershman y Stelarc» ${ }^{27}$. La organización del libro deja entrever una de las principales hipótesis de las que Shanken se vale para proponer una revisión de la historia del arte canónica, a saber, que la innovación técnica y el uso de tecnologías emergentes como medios creativos tuvieron continuidad en la historia del arte occidental, desde la creación de la pintura al óleo hasta el desarrollo de entornos virtuales interactivos y el arte

24. E. Shanken, “Contemporary Art and New Media..., op. cit.

25. E. Shanken, Art in the Information Age..., op. cit.

26. E. Shanken, Inventar el futuro..., op. cit., 112.

27. E. Shanken, Inventar el futuro..., op. cit., 112. 
telemático ${ }^{28}$. Sin embargo, como argumentamos en el apartado precedente al cuestionar la teoría de Bishop en torno a la noción de desmaterialización, esta clase de enfoques transversales no han sido frecuentes en muchas de las teorías del arte extensamente difundidas:

Por ejemplo, Art Since 1900 (2004) es un texto canónico sobre el arte moderno y contemporáneo escrito por Hal Foster, Rosalind Krauss, Yve-Alain Bois y Benjamin Buchloh, el que podría considerarse como el principal grupo de historiadores del arte contemporáneo de Estados Unidos, sino del mundo. Sin embargo, es tal el grado de desconocimiento (o de hostilidad) de sus autores respecto a cualquier tipo de arte que utilice medios tecnológicos, que ignoran incluso los mayores hitos de los discursos de la historia del arte de los nuevos medios, como Billy Klüver y E.A.T. Si Klüver y E.A.T. no le resultan familiares al lector, no es por su culpa, sino que más bien eso demuestra el problema. ${ }^{29}$

Otra de las tensiones entre la escena del arte contemporáneo y las poéticas electrónicas fue revelada en 2010, cuando en Art Basel Shanken organizó una mesa redonda titulada Contemporary Art and New Media: Towards a Hybrid Discourse. Los invitados a disertar sobre el tema fueron Nicolas Bourriaud, Peter Weibel y Michael Grey, tres referentes de ambos circuitos. Según Shanken, una muestra evidente de la escisión entre los dos mundos es que Weibel y Bourriaud no se conocían, a pesar de que el primero es uno de los artistas y teóricos más significativos de la escena del arte y la tecnología, mientras que el segundo es un reconocido curador y académico en el terreno del arte contemporáneo dominante ${ }^{30}$. Las discusiones devenidas allí fueron productivas, dado que pusieron de manifiesto algunos de los prejuicios que muchas veces recaen sobre las poéticas electrónicas. En línea con la perspectiva esbozada en su libro Estética relacional, Bourriaud sostuvo que las tecnologías influyen de manera indirecta en el desarrollo de las prácticas artísticas, en tanto la irrupción de cualquier nueva tecnología inaugura modelos de pensamiento hasta entones inexistentes: la fotografía repercutió entre los artistas impresionistas, configurando un nuevo modo de ver el mundo. Para el autor francés, la computadora o la red no constituyen medios en sí mismos, sino herramientas empleadas en el contexto de la era postmedia. Luego de la exposición de Bourriaud, Weibel retomó el impacto ejercido por la fotografía en la pintura del impresionismo, para recordar que a su vez el campo fotográfico produjo sus propias obras. En pocas palabras, sería un error dejar de considerar a las tecnologías como medios legítimos destinados a la creación artística. Shanken sintetizó la contradicción de esta manera: «Peter Weibel retomó astutamente la distinción de Bourriaud entre influencias directas e indirectas y advirtió la incongruencia de valorar la influencia indirecta de la tecnología al tiempo que se ignora el uso directo de la tecnología como medio artístico de pleno derecho» ${ }^{31}$. De acuerdo a su opinión, las argumentaciones de Bourriaud acaban legitimando el discurso del arte

28. E. Shanken, Art and Electronic Media, Londres, 2009.

29. E. Shanken, Inventar el futuro..., op. cit., 114-115.

30. E. Shanken, Inventar el futuro..., op. cit., 117.

31. E. Shanken, Inventar el futuro..., op. cit., 120. 
contemporáneo hegemónico, el cual frecuentemente se ha mostrado reacio a aceptar como parte de su historia a muchas de las prácticas artísticas que incorporan medios emergentes. Este punto condujo al autor a concluir que «(...) un relato del arte contemporáneo en el que los nuevos medios sean un componente central requiere una historia diferente que incluya una reevaluación de los hitos principales» ${ }^{32}$.

Un relato planteado en estos términos supondría analizar, por ejemplo, la obra de Sol LeWitt en relación a otros proyectos contemporáneos, los cuales, a través del uso explícito de las tecnologías, también atendieron a las lógicas matemáticas que gobernaban la propuesta artística. Entre ellos, las obras algorítmicas de Manfred Mohr, como su animación por computadora titulada Cubic Limit, realizada entre 1973 y 1976 investigando las posibilidades de la combinatoria programada, o las variaciones geométricas de Vera Molnar, quien a partir de 1968 comenzó a utilizar el ordenador para diseñar dibujos lineales que originalmente eran ploteados y, años después, pasaron a ser impresos. Independientemente de las diferencias en los medios y herramientas utilizados por cada uno de los artistas, en los tres casos las prácticas artísticas demuestran una carácter sistémico, asociado al devenir de obras abiertas y procesuales fundadas en las relaciones dinámicas establecidas entre sus partes, así como en las estructuras que subyacen en determinados comportamientos perceptibles.

En el contexto argentino, la historia del arte generativo por computadora podría hibridarse con sus tempranas manifestaciones en el campo pictórico. En 1960, Miguel Ángel Vidal y Eduardo Mac Entyre redactaron el «Manifiesto de arte generativo», donde describieron a la pintura generativa como aquella capaz de engendrar secuencias ópticas mediante un desarrollo generado por una forma. Ya en ese entonces detectaban la relación entre la pintura generativa y nociones vinculadas a la tecnología, como la fuerza y la energía. Incluso la definición del término «generador» que los artistas proporcionan en su escrito, «dícese de la línea o la figura que por su movimiento engendra respectivamente una figura o un cuerpo geométrico» ${ }^{33}$, evoca un grado de autonomía de los elementos plásticos con respecto al plano, coincidente con la difundida caracterización de las prácticas artísticas generativas según Philip Galanter ${ }^{34}$. Su teoría sostiene que los artistas generativos ceden el control de la obra a un sistema que opera con cierta independencia de acuerdo a una serie de reglas e instrucciones preestablecidas. Mientras que la pintura generativa nace de la vibración, el giro y el desplazamiento de los elementos plásticos que se originan progresivamente despegándose de la superficie plana que los contiene, el arte generativo basado en las tecnologías digitales es creado por medio de algoritmos que constituyen la arquitectura de datos a partir de la cual la obra se autogenera.

32. E. Shanken, Inventar el futuro..., op. cit., 118.

33. E. Mac Entyre y M.A. Vidal, "Manifiesto de arte generativo”, 1960. En línea en: http://www.miguelangelvidal.com.ar/manifiesto.html. [Consulta: 02.06.19].

34. P. Galanter, "What is Generative Art: Complexity Theory as a Context for Art Theory”, 2003. En línea en: http://www.philipgalanter.com/downloads/ga2003_paper.pdf. [Consulta: 02.06.19] 
No obstante, el relevamiento de puntos de convergencia entre ambas historias no debe limitarse a un relato lineal de desarrollos encadenados que descuide las especificidades de sus respectivas prácticas. Söke Dinkla expuso críticas oportunas hacia la homologación sin distinciones entre las primeras obras participativas de los años cincuenta y sesenta, como ambientaciones, performances y happenings, y el surgimiento del arte interactivo. Frente a las ideas de Regina Cornwell y Erkki Huhtamo, quienes postularon que el rol activo del público en las obras interactivas es una derivación directa de las reconfiguraciones instauradas con el happening y otras manifestaciones del arte participativo -Allan Kaprow, Robert Rauschenberg, Yoko Ono, Fluxus y Situacionismo, entre otras-, Dinkla propone atender también a las diferencias introducidas por las características privativas de la interactividad. Aunque los trabajos de Jeffrey Shaw, Lynn Hershman Leeson, Peter Weibel y Bill Seaman reconocen nexos con el arte participativo, las producciones de Myron Krueger o David Rokeby resultan de sus métodos orientados hacia la investigación estrictamente tecnológica ${ }^{35}$. Por otro lado, si el happening se vincula con el teatro experimental y su distanciamiento de la estructura dramática clásica, gira en torno a la relación (neo) vanguardista arte/vida y sus artistas aún se encuentran presentes en la obra, los proyectos interactivos no proceden de géneros artísticos determinados, reemplazan el vínculo arte/ vida por el par arte/tecnología, sus autores desaparecen de la escena al ser sustituidos por procesos automatizados y el material artístico de las obras es el diálogo entre el programa y el usuario ${ }^{36}$. Las particularidades de unas y otras prácticas también tienen que ser consideradas al momento de elaborar un nuevo relato sobre el arte contemporáneo.

La construcción de una nueva historia del arte -o bien, de su lado B- exige la adopción de herramientas epistemológicas que permitan complementar conocimientos de la teoría e historia del arte con determinadas nociones sobre ciencia y tecnología. Y es allí donde reside uno de los mayores desafíos. En la introducción de New Media in the White Cube and Beyond: Curatorial Models for Digital Art, Christiane Paul caracteriza al paradigmático cubo blanco como un espacio que presenta evidentes limitaciones para albergar propuestas performáticas o que, aun cuando no lo sean, solicitan espacios que no se limiten a la contemplación estética:

Los nuevos medios nunca podrían ser entendidos solamente desde la perspectiva de la historia del arte: la historia de la tecnología y las ciencias mediales desempeñan un rol igualmente importante en la formación y recepción de las artes. Los nuevos medios requieren de una alfabetización mediática. ${ }^{37}$

35. S. Dinkla, "From Participation to Interaction: Toward the Origins of Interactive Art", en L. Hershman Leeson (ed.), Clicking in: Hot Links to a Digital Culture, Seattle, 1996, 282.

36. S. Dinkla, "From Participation...”, op. cit., 288-289.

37. Ch. Paul, New Media in the White Cube and Beyond, Berkeley, 2008, 5. Traducción propia. «New media could never be understood from a strictly art-historical perspective: the history of technology and media sciences plays an equally important role in this art's formation and reception. New media art requires media literacy.» 
Al igual que es preciso reformular el contexto adecuado para exhibir toda clase de poéticas electrónicas, cabe repensar las perspectivas teóricas desde las cuales dichas obras son abordadas. La alfabetización referida por Paul en el pasaje citado implica la instrucción de nuevos espectadores, pero también -en primer lugar- supone la formación de historiadores del arte, críticos y curadores, quienes además de analizar las obras estéticamente, comprendan su propuesta técnica y puedan desentrañar comportamientos a menudo complejos.

\section{Conclusiones}

El análisis desarrollado a lo largo del artículo respondió a un doble propósito. Por un lado, hemos reconstruido el estado de la cuestión relativo a la historiografía del arte contemporáneo, con el objetivo de rastrear las principales teorías que, durante los últimos años, dieron fe del fenómeno designado en el segundo apartado del escrito como «desconexión historiográfica», dado por una cierta exclusión de las prácticas artístico tecnológicas por parte de la historia del arte dominante. Las obras que emplean tecnologías de manera preeminente comenzaron lentamente a delinear un ámbito alejado del mundo del arte contemporáneo. Uno y otro fueron delineando nichos separados provistos de sus propias lógicas de producción, difusión, crítica e investigación, en un proceso iniciado en los años sesenta en paralelo a la irrupción del arte contemporáneo, e instituido hacia la década del noventa cuando el boom digital aceleró la conformación de la esfera de las artes electrónicas como un circuito autónomo. La instauración de ambas escenas diferenciadas proporcionó a unas el adjetivo de «tecnológicas», mientras que otras acabaron configurando un circuito independiente clasificado como «arte contemporáneo», si bien es evidente que en los dos casos se trata de prácticas artísticas contemporáneas y muchas de las obras que asiduamente integran el segundo conjunto también podrían ser consideradas tecnológicas: ya sea porque se parta de la premisa de que toda obra emplea tecnologías - un extenso debate que en ocasiones ha conducido a considerar que la pintura, el dibujo y otras disciplinas tradicionales también utilizan tecnologías, aun cuando éstas no consistan en tecnologías electrónicas y/o digitales-, o bien porque se entienda que las obras constituyen modos de decir y de hacer que asumen su propio tiempo técnico, en cuyo caso podrían ser calificadas como poéticas tecnológicas ${ }^{38}$.

Por otra parte, en correspondencia con el planteo anterior, hemos argumentado en el tercer apartado que es posible sentar las bases de una nueva historia del arte contemporáneo, reconsiderando determinadas nociones que han sido acuñadas para definir a este campo -entre ellas, la categoría de desmaterialización-, pero ampliando sus horizontes de cara a examinar asimismo las resonancias de dichos conceptos en obras tecnológicas. Las tentativas de reescritura de la historia señaladas en el cuarto apartado impulsan a incorporar en sus relatos a artistas y obras que no han sido frecuentemente incluidos en las narrativas hegemónicas, aunque sus búsquedas creativas y conceptuales se encuentran cabalmente alineadas

38. C. Kozak (comp.), Poéticas/politicas tecnológicas en Argentina 1910- 2010, Paraná, 2014. 
con aquellas encauzadas por artistas contemporáneos ajenos a la exploración tecnológica (quienes sí fueron reconocidos por la historia canónica).

Un aspecto que en este artículo no ha sido abordado y que, en consecuencia, queda pendiente para futuros trabajos, consiste en el estudio de potenciales iniciativas institucionales - museo, centro cultural u otra plataforma que responda a un formato acorde con las revisiones contemporáneas de los ámbitos tradicionales de producción y exposición-, dedicadas de manera íntegra y sistemática a la investigación y difusión de las poéticas electrónicas y sus relaciones con el arte contemporáneo. O bien, un espacio consagrado a la escena del arte contemporáneo y sus intercambios con las poéticas electrónicas inscritas en ella. Un programa de estas características debería también propiciar la intersección entre la labor académica y el trabajo artístico tecnológico, así como la comunicación con diferentes iniciativas institucionales que de modo parcial se encuentran abocadas a los cruces entre el arte y la tecnología. De esta manera, las nuevas historiografías del arte contemporáneo no solo lograrían articularse desde la producción teórica, sino también configurar imaginarios alternativos desde la propia gestión de la cultura.

Las estrategias enunciadas evitan segregar a las prácticas contemporáneas en función de criterios ceñidos a los medios, herramientas y soportes empleados, razones carentes de sentido desde el momento en que la multiplicidad de formatos y lenguajes es, precisamente, una de las características medulares del arte de nuestra era. Después de todo, la reformulación de la historia hegemónica permite al fin levantar los límites que proyectan a las poéticas electrónicas separadas del terreno del arte contemporáneo. Cincuenta años después de la rotunda expansión del campo artístico, las tecnologías ya no tienen por qué ser imperiosamente exaltadas, ni encarnar una frontera que condene a sus prácticas a vivir en el exilio. 